\title{
Are astrocytes executive cells within the central nervous system?
}

\author{
¿Son los astrocitos células ejecutivas dentro del Sistema Nervioso Central? \\ Roberto E. Sica ${ }^{1}$, Roberto Caccuri', Cecilia Quarracino ${ }^{1}$, Francisco Capani ${ }^{1}$
}

\begin{abstract}
Experimental evidence suggests that astrocytes play a crucial role in the physiology of the central nervous system (CNS) by modulating synaptic activity and plasticity. Based on what is currently known we postulate that astrocytes are fundamental, along with neurons, for the information processing that takes place within the CNS. On the other hand, experimental findings and human observations signal that some of the primary degenerative diseases of the CNS, like frontotemporal dementia, Parkinson's disease, Alzheimer's dementia, Huntington's dementia, primary cerebellar ataxias and amyotrophic lateral sclerosis, all of which affect the human species exclusively, may be due to astroglial dysfunction. This hypothesis is supported by observations that demonstrated that the killing of neurons by non-neural cells plays a major role in the pathogenesis of those diseases, at both their onset and their progression. Furthermore, recent findings suggest that astrocytes might be involved in the pathogenesis of some psychiatric disorders as well.
\end{abstract}

Keywords: astrocytes; physiology; central nervous system; neurodegenerative diseases.

\section{RESUMEN}

Evidencias experimentales sugieren que los astrocitos desempeñan un rol crucial en la fisiología del sistema nervioso central (SNC) modulando la actividad y plasticidad sináptica. En base a lo actualmente conocido creemos que los astrocitos participan, en pie de igualdad con las neuronas, en los procesos de información del SNC. Además, observaciones experimentales y humanas encontraron que algunas de las enfermedades degenerativas primarias del SNC: la demencia fronto-temporal; las enfermedades de Parkinson, de Alzheimer, y de Huntington, las ataxias cerebelosas primarias y la esclerosis lateral amiotrófica, que afectan solo a los humanos, pueden deberse a astroglíopatía. Esta hipótesis se sustenta en hallazgos que demostraron que la muerte neuronal que en ellas ocurre es debida al compromiso de células no-neuronales que juegan rol principal en su iniciación y desarrollo. Más aún, observaciones recientes señalan que los astrocitos podrían estar implicados en la patogenia de algunas enfermedades psiquiátricas.

Palabras clave: astrocitos; fisiología; sistema nervioso central; enfermedades neurodegenerativas.

The physiological properties of astrocytes have been the subject of comprehensive reviews written in the last few years ${ }^{1}$, while other authors have highlighted the role that astrocytes play in the development of diseases previously attributed solely to neurons ${ }^{2,3}$.

Our aim is to present a brief overview of the interplay between astrocytes and neurons in normal and pathological conditions within the central nervous system (CNS).

\section{ASTROCYTES IN PHYSIOLOGY}

Several investigations have described the capabilities of the astrocytes in handling part of the workload of the CNS. They modulate the myelinating capacity of the oligodendrocytes $^{4}$, the synaptic cross-talk amongst neurons ${ }^{5}$, the homeostasis of the CNS extracellular medium 6 , the initiation of the inflammatory and immune responses ${ }^{7}$ and the building up of barriers between normal and damaged tissues ${ }^{8}$. Astrocytes can behave like phagocytic cells ${ }^{9}$, they sense the partial pressure of $\mathrm{O}_{2}$ within the $\mathrm{CNS}^{10}$ and they can transform into neurons when the brain is in need of rebuilding damaged functional neural networks contributing to nervous tissue repair ${ }^{11}$. Therefore, we believe that the astrocyte deserves to be considered, in its own right, one of the main players orchestrating fundamental functions within the CNS.

Since Ramon y Cajal's neuron theory was accepted in 1889, most researchers thought that neurons were the most valuable cells in the functions performed by the CNS. If we critically analyze their properties, the foremost is their capacity to convey messages at high speed by propagating action potentials (APs) that travel through their dendrites, bodies

1 Universidad de Buenos Aires, Escuela de Medicina, Instituto de Investigaciones Cardiológicas, Buenos Aires, Argentina. 
and axons membranes. The electrical activity is of such paramount importance for neurons that it consumes $75 \%$ of the brain cortical energy; one fifth of it is employed to sustain APs, another fifth accounts for the maintenance of the membrane resting potential, and the remainder is consumed in synaptic dynamics. "Housekeeping", a term coined by Attwell's group, meaning the non-signalling tasks performed by the neurons (such as proteins shuttle and turnover, axoplasmic flows and other intracellular processes), consumes only $25 \%$ of the total energy. Knowing that the brain cortical energy has been estimated in $27.2 \mu \mathrm{mol} \mathrm{ATP} / \mathrm{g} / \mathrm{min}$, neuronal signal activity requires $20.41 \mu \mathrm{mol} \mathrm{ATP} / \mathrm{g} / \mathrm{min}$, more than twice the energy consumed to carry out the intraneuronal metabolic process$\mathrm{es}^{12}$. The conclusion that can be drawn from these observations is that the function prioritized by neurons is signalling.

In order to maintain the integrity of the body, most changes within the environment of a living being need a response that, in special circumstances, must be achieved effectively and quickly, such as happens with the reflex responses that are below the conscious level. These responses occur in a milliseconds time scale, which has always been regarded as an exclusive domain of neurons. However, some studies have found that astrocytes can release, by exocytosis, the gliotransmitter glutamate into the synaptic cleft in the same time scale that neurons employ, suggesting that astrocytes may have a role in the fast synaptic activity, matching the capacities of the neurons in this aspect ${ }^{13}$. This observation indicates that astrocytes may have a role in the elaboration of the reflex responses.

\section{Neuron-astrocyte dynamics}

Further reasoning leads to the question: What drives the functions of neurons when a response has to be elaborated to adapt the conditions of the self to the environs, differentiating the right response amongst many possibilities? We know that the synaptic transmitters carry information from one neuron to another that receives the message through ionotropic receptors. We also know that there is protein traffic within the neuron's body that is activated by second messengers that are put to work by the stimulation of metabotropic receptors located on the cell and neurite membranes. The post-synaptic neuron receiving the information from the presynaptic neuron will repeat the cycle stimulating other neuron until the desired goal is achieved. But, are the neurons free to deliver these types of messages just by themselves?

Synaptic activity is modulated by astrocytes, which enhance or diminish the synaptic strength by delivering gliotransmitters and transmitter transporters ${ }^{14}$ while providing neurons with glucose and $\mathrm{O}_{2}$ and adapting the blood flow to their requirements, thus establishing a very close relationship with them. Moreover, astrocytes are the source of not only glucose and $\mathrm{O}_{2}$, but also of other metabolites such as lactate, fatty acids and trophic factors that sustain neuronal health ${ }^{15}$.
Observations recently reported suggest that astrocytes may have an even more intimate relationship with neurons by establishing contact with them through gap junctions $(\mathrm{GJ})^{16}$ that allow both types of cells to exchange molecules and ions, modifying their metabolism and the expression of their genes ${ }^{17}$.

\section{Anatomic phylogenetic remarks}

In humans, glial cells are the largest family of cells within the CNS. Friede was the first to compare the numbers of glia and neurons and found that, in humans, glia outnumber neurons by 1.48, a factor known in the literature as the glia/neuron ratio ( $\mathrm{G} / \mathrm{Nr}$ ratio) ${ }^{18}$. In species considered phylogenetically below humans, those ratios were smaller; for instance in frogs it was 0.25 , chickens 0.46 , mice 0.36 , rabbits 0.43 , pigs 1.20 , cows 1.22 and horses 1.23 . The higher human $\mathrm{G} / \mathrm{Nr}$ ratio was initially considered a peculiarity of the human species ${ }^{19}$.

However, in contrast to those findings, Hawkins et al. found a $4.54 \mathrm{G} / \mathrm{Nr}$ ratio in the cortex of the fin whale ${ }^{20}$, suggesting that the $\mathrm{G} / \mathrm{Nr}$ ratio is not related to the phylogenetic position of the species. Moreover, by estimating the number of neurons within the brain of several species they concluded that the density of neurons decreases as the volume of the brain increases. So, the number of neurons in the brains of mice is $142,000 / \mathrm{mm}^{3}$, in humans the figure was estimated to be about 9,500 neurons $/ \mathrm{mm}^{3}$, while the brain cortex of the fin whale and the Indian elephant has about 7,000 neurons $/ \mathrm{mm}^{3}$, a lower value than in humans ${ }^{21,22}$.

One possible explanation for the reduced number of neurons in highly-developed phylogenetic species is that the neurons are larger in size, assembled with a greater number of dendrites and thicker axons streaming through bigger territories. These morphological neuronal changes parallel the behaviour of the astroglia, which become larger in size, both in humans and in apes, with thicker and longer processes depicting bigger domains encompassing up to 2 million synapses in humans compared to, for example, a maximum of 120,000 in mice.

In summary, it appears that the composition of the cells in the cerebral cortex does not explain the different behaviours between species. Hence, human intellectual properties cannot be attributed either to an increased number of neurons or to a larger number of astrocytes.

Herculano-Houzel's findings ${ }^{23}$ support what has been stated above. The author found that the number of astrocytes is not greater than the number of neurons in the human brain cortex, but their sizes are larger when compared with other species. Herculano-Houzel's observations are logical for at least three reasons. The first, given by the author, is that despite the fact that protoplasmic and fibrous astrocytes inhabit the brain cortex, only protoplasmic astrocytes are related to neurons and their synapses. The second is that most of the authors who have suggested that glial cells outnumber 
the neurons refer to glia as a generic term encompassing all types of glia. Finally, the third, and perhaps the most convincing reason from our point of view, is that protoplasmic astrocytes residing within the gray matter of the brain cortex are organized in domains that do not overlap. This means that a single astrocyte contacts a group of neurons and their synapses, suggesting that in this region of the encephalon, the number of neurons must be larger than the number of astrocytes. In fact, Pelvig et al. have estimated that the percentage of astrocytes within the human cortex is only $20 \%$ of all glial cells residing in that structure ${ }^{24}$.

Therefore, it seems that the role of astrocytes in the physiology of the brain cannot be based on the frequency of their presence but in the function that they accomplish as neuronal partners.

\section{A thought about thinking}

Decision-making and abstract thoughts are well-defined characteristics of primates ${ }^{25}$. These higher mental functions encompass the action of thousands of neurons within the cerebral cortex immersed in a complex cross-talk that may last several seconds, even minutes, until a determination fitting the request is made at the conscious level.

Being human the species that can carry out complex abstract thinking, a property partially shared with the great apes, it is possible to hypothesize, that this capacity is partially related to the peculiar relationship that neurons have with the astrocytes that are intimately related to them.

We know, albeit incompletely, the role of each cell type within the CNS: neurons receive and carry messages; oligodendrocytes constitute the myelin factory, microglia guard the CNS and, finally, astrocytes support the oligodendrocytes $^{26}$ and mix intimately with the neurons and their synapses $^{5}$, up to the point that currently a synapse is considered to have three components, pre-synaptic, post-synaptic and astrocytic: the tripartite synapse ${ }^{27}$.

Evolution has provided complexity to living beings within the animal kingdom with the aim of adapting them to their environments. To obtain this objective, the being has to explore and judge the state of its environment. For these purposes it has to employ a particular tool to be able to obtain information about its surroundings, which has to be processed and translated into an adequate response. This tool is the nervous system; the first hints of its appearance were present in the sponge, which has no neurons but has a set of post-synaptic scaffolding genes pre-announcing them ${ }^{28}$. After the divergence of sponge and eumetazoan, this last lineage developed transmembrane receptor genes, such as the glutamate receptors family found at the post-synapse of the most developed species, where recognizable nervous structures can be found ${ }^{29}$.

There is almost universal agreement that the cornerstones supporting evolution are genetic changes and activitydependent mechanisms ${ }^{30}$. Perhaps the peculiar relationship between astrocytes and neurons that evolution has developed within the animal kingdom should be added.

If astrocytes are involved in cognitive functions, which is their role and which is the role of neurons in these activities? Possibly the answer to this question resides in the connection that neurons and astrocytes establish at the tripartite synapse $^{16,31}$.

It is known that in mammals, particularly in primates, synapses can undergo plastic changes both by dendritic branching and by increasing the number of dendritic spines, allowing higher synaptic volume and strength; these modifications being more notable in the neocortex of larger brains $^{32,33}$. However, there are constraints that limit the anatomic growth of the brain ${ }^{33}$. To overcome them the brain develops plastic modifications not only at the synaptic level, but also by connecting different cortical regions, creating new circuits that will work in a coordinated manner with the aim of obtaining a desired purpose. Growth of new synapses can even occur in long-term memory processes; changes that recruit molecules swiftly by altering the genetic pattern of the structure and triggering novel biochemical pathways that will introduce modifications within the neurons' functions.

Higher mental functions involve the activation of transmitter receptors, either ionotropic or metabotropic, located pre- and post-synaptically. Transmitter receptors can be found in every organism with a nervous system, but synapses become more numerous and more complex in more phylogenetically-evolved animals, allowing them to develop an increased behavioural capacity. In mammals this capacity is framed within an expanded nervous system that undergoes regional specialization, a characteristic that permits these organisms to be more flexible and plastic when responding to environmental changes. An interesting question is whether brain regional specialization means synaptic specialization as well and, if so, which parts of the synapse are responsible for the development of that property. A partial answer to this enquiry may be that the changes of the synaptic proteoma are due to modifications of the expression of genes involved in synaptic activity ${ }^{34}$; however, this might not be the only reason justifying the synaptic changes.

\section{Astrocyte synaptic involvement}

When analyzing the structure of the synapses, particularly in mammals and mainly in primates, it is evident that not only pre- and post-synaptic neuronal structures are to be found, but also the branches of the astrocytes that enwrap the whole synapse, suggesting that astrocytes may play a crucial role in the information processing occurring at the synapses by establishing a fluent cross-talk with neurons.

Astrocytes do not produce propagated membrane potentials, but they can sustain resting membrane potentials of about $90 \mathrm{mV}$ due to the dense presence of $\mathrm{K}^{+}$channels. Also, they are able to alter their membrane excitable state by changing their $\mathrm{Ca}^{++}$load, which can propagate 
within an astrocytic net at different speeds according to the species ${ }^{35}$. Human astrocytes are able to propagate $\mathrm{Ca}^{++}$ signals at a faster rate than other species, the mean speed being $43.4 \pm 4.7 \mu \mathrm{m} / \mathrm{s}$, while in rodents, for example, the speed is $8.6 \pm 0.6 \mu \mathrm{m} / \mathrm{s}$.

Excitability may be quite specific, as there are micro compartments within the astrocyte where different amounts of $\mathrm{Ca}^{++}$can be stored, allowing particular excitability states of discrete territories within the cell. This uniqueness is important as the region of the astrocyte submitted to this change sends its message to the processes born in the membrane belonging to that particular micro compartment, which will contact and influence the membranes of other cells with which the astrocyte is associated, including neurons and their synapses.

Astrocytes are sensitive to the state of the synapses they reach. They may enhance or halt their activity according to the circumstances. There is feedback correspondence between the synapses and the astrocytes, so astrocytes increase their $\mathrm{Ca}^{++}$load and the value of their membrane potential when the synapses with which they are related are activated. Such behaviour allows astrocytes to modulate the neuronal and the astrocytic web in which they are situated by the generation of transmitters that are able to act at the neuronal synaptic receptors as well as on extra-synaptic receptors ${ }^{36,37}$.

Three main transmitters are released by the astrocytes; two are excitatory: glutamate and D-serine. The latter is converted to L-serine by the serine racemase enzyme; L-serine is an essential co-agonist to the glycine site of the NMDA receptor acting as a glutamate co-transmitter. The third transmitter, ATP, which changes to ADP, AMP and, finally, to adenosine, inhibits the glutamate release from neighbouring pre-synaptic neurons, down regulating the excitatory synaptic transmission at several neuronal locations simultaneously.

These properties of astrocytes allow them to coordinate the activity of a whole neuronal pool.

Astrocytes are indeed capable of being excited by $\mathrm{Ca}^{++}$ waves within their cytoplasm. These waves can be transmitted from astrocyte to astrocyte through their GJs while releasing ATP through unopposed hemichannels. The ATP will act, as well, on astrocytic purinergic receptors whose activity will increase the intracellular $\mathrm{Ca}^{++}$, contributing to the expansion of calcium waves within astrocytes. This behaviour embraces a large population of astrocytes that will influence the activity of the neurons they contact. Balancing the production and release of transmitters these cells contribute to poise the excitability state of the neuronal pool to which they are related ${ }^{38}$.

Other means by which the astrocytes modify the excitability state of the neurons they contact is through the control of $\mathrm{K}^{+}$concentration within the external milieu due to the presence of potassium channels in their membranes. Kir4.1 potassium channel, a member of the inward rectifying $\mathrm{K}^{+}$ channels family, is responsible for maintaining the membrane potential of astrocytes and for buffering the extracellular $\mathrm{K}^{+}$ under normal conditions. The astrocytes' production of glutamate transporters EAAT1 (GLAST) and EAAT2 (GTL-1) is another instrument by which they are able to regulate the excitability state of the neurons. These transporters are built up within the astrocytes and transferred to the extra-cellular medium where they pick up the glutamate employed in the cross-talk amongst neurons and between neurons and astrocytes, carrying it into the astrocytes where it will be transformed into glutamine through the action of the glutaminesynthetase enzyme. In turn, glutamine will be transported back to the pre-synaptic neurons where it will be hydrolyzed by the glutaminase enzyme, generating glutamate.

\section{Astrocyte domains and subtypes}

Protoplasmic astrocytes are plastic cells, devoted entirely to the neurons they contact. Their shape and appearance vary along the CNS in relation to the neuronal type they interact with. They are organized in specific territories with different anatomical and metabolic characteristics and different gene expression profiles, in harmony with the neuron's requirements ${ }^{39}$. Examples of this behaviour are the Müller cells in the retina, which are adapted to the needs of the neurons within that structure and the Bergmann cells in the cerebellum, which are partners of the Purkinje neurons.

Therefore, it becomes reasonable to assume that the astrocytes that influence a particular population of neurons devoted to a specific function constitute a singular population, only devoted to those types of cells ${ }^{2,40}$; in this regard they should have their architecture and a metabolism particularly suited to the sustenance and control of those neurons' needs and activities.

These singular qualities bring up the concept of "unique populations of astrocytes" meaning that astrocytes devoted to a pool of neurons performing a specific task have peculiarities not shared by other populations of astrocytes connected with other type of neurons performing other functions. This concept recently received support from Martin et al. who found that medium spiny neurons (MSN) belonging to two different circuits -the direct and indirect pathways of the basal ganglia- are associated with a discrete subpopulation of astrocytes that selectively respond to one MSN circuit, but not to the other and vice versa ${ }^{41}$.

In humans and chimpanzees it is possible to find other two kinds of astrocytes not present in other species: the varicose astrocytes, residing in layers 5 and 6 of the neocortex, extending long processes characterized by regularly spaced varicosities that contact the neuropil and the vasculature; and the interlaminar astrocytes located mainly at the supragranular cortex that extend tortuous processes to the pial surface and the cortical layers in a sort of columnar fashion terminating mainly at the neuropi ${ }^{42}$. These types of astrocytes might play a crucial role in orchestrating the harmonic and organized activity of the protoplasmic astrocytes. They 
do not have domains, rather their processes cross different and separated protoplasmic astrocytes domains, contacting not only the neuropil and the vessels, but the fibres of the residing protoplasmic astrocytes as well, possibly establishing a peculiar cross-talk with them. Their functions are poorly known at present and a matter of current debate.

It is worth mentioning once more that the protoplasmic astrocyte domains in humans are the most extensive in Nature, comprising the largest number of synapses in a living being $^{43}$. Perhaps this anatomical design, which in the future will probably include the varicose and the interlaminar astrocytes as well, can be interpreted as the structural basis of a more complex function - performed by what can be named neuron-astrocyte unit of work - with greater powers of computational capacities than in other species, by coordinating the activity of a large pool of neurons and synapses.

Fibrous astrocytes are not organized in domains. In humans they are larger than in lower phylogenetic species and their processes are oriented in the direction of the axons with which they are related. They establish contact with processes of other fibrous and protoplasmic astrocytes and with the vessel walls located in their neighbourhood.

As far as we currently know, fibrous astrocytes are capable of sharing information with other fibrous and protoplasmic astrocytes through the GJs existing at their contacts by employing travelling $\mathrm{Ca}^{++}$waves and exchanging small molecules. Also they participate in the glutamate metabolism by expressing glutamate membrane receptors and transporters. An interesting structural detail of these cells is that they send fine processes to the nodes of Ranvier situated at the axons with which they are related, establishing a particular relationship that shall be discussed later.

\section{The Neuron coding system}

\section{Protoplasmic astrocytes participation}

The information managed by the CNS is mediated through the employment of a code that it is built up by the timing and the frequency of the APs deployed by the neurons, which ultimately travel through their axons. Therefore, it is possible to say that the language spoken by neurons is the combination of rhythm and timing of their APs discharge.

However, which factors influence the neurons to establish the timing of the APs and their rhythm of discharge? Some of them are known, consisting of the following neuronal features: a) amount of transmitter released by the presynaptic axon, b) number of available post-synaptic neuron receptors, c) active duration of the transmitter within the synaptic cleft, d) kinetics of the transmitter's transporter within the synaptic cleft, e) ionic composition of the synaptic medium, f) functional state of the $\mathrm{Na}^{+} / \mathrm{K}^{+}$Atepase pump and other ion channels at the pre-synaptic axon's terminals and post-synaptic dendrites and neuron membranes. From the astrocytic side, astrocytes release transmitters and transporters already mentioned. They simultaneously balance the
$\mathrm{K}^{+}$concentration within the external medium and increase the concentration of arachidonic acid and $\mathrm{Ca}^{++}$in the astrocytic end-feet that control cerebral microcirculation. This is accomplished through the arachidonic acid metabolites prostaglandin E2 and epoxyeicosatrienoic acids that determine arteriole dilation, while 20-hydroxyeicosatetraenoic acid induces arteriole constriction. These changes appear in synapses, neurons and astrocytes integrating a network with a defined purpose. However, despite astrocytes being an essential part of the synapses, their contribution to synaptic changes are only partially known; in spite of this, in the last few years, their role in complex behaviours, such as breathing, sleep and information processing has been demonstrated.

When synaptic signals arise, they awaken the activity of the astrocytes to which they are related. By the time the astrocytes learn that a neuronal signal has reached the synapses that are under their control, they sense the synaptic characteristics developed at the site and modulate the synaptic activity. This either enhances or diminishes their strength by secreting excitatory glio-transmitters that will increase the synaptic efficacy or ATP that will lessen the synaptic excitatory state, and transporters that will reduce the amount of transmitters at the synaptic cleft lowering the synaptic strength ${ }^{44}$. This will determine the timing and rate of discharge of the neuronal post-synaptic potentials and, consequently, the meaning of the message. These changes will also be transmitted to other astrocytes related to neurons that have similar functions and with which the former activated astrocytes contact through their GJs. This builds up a coordinated response within the net that they constitute that will be translated into the characteristics of the code that the post-synaptic neurons will send to the next synaptic station. If this is the case, it has to be accepted that astrocytes are cells that strongly contribute to the establishment of the neuronal response. Within this context an interesting possibility might arise: the population of astrocytes involved in the activated net may perfect their response by employing oscillations of their $\mathrm{Ca}^{++}$load and producing specific $\mathrm{Ca}^{++}$waves that will reach the astrocytes enwrapping the activated synapses modulating, in a coordinated manner, the synaptic response, hence compelling the post-synaptic neurons to discharge EPs employing a code that is partially conditioned by the astrocytes' behaviour.

Therefore, the relationship among the pre-synaptic neurons, the post-synaptic-neurons and the astrocytes will define the strength of the synapses and the timing and frequency of the APs that will be carried by the post-synaptic axons to reach other neurons, transmitting a message that will be analysed once more in these new synapses by employing the same mechanism.

\section{Fibrous astrocytes participation}

Another fact that deserves contemplation is one of the anatomical features that characterizes the anatomy of the fibrous astrocyte: this is the process that it sends to the node 
of Ranvier of the axon under its surveillance. The nodes of Ranvier are small, complex regions, about $1 \mu \mathrm{m}$ long, along myelinated nerve fibres where the myelin is interrupted allowing the axon membrane to be in contact with the extracellular medium. Within this area devoid of glial sheaths there is a high concentration of voltage gated $\mathrm{Na}^{+}$channels located at the axonal membrane and $\mathrm{K}^{+}$channels situated on either side of the node, at the juxtaparanodal regions. This anatomical design allows saltatory conduction, increasing the speed of the travelling AP along the myelinated axons. For proper function the node needs a high density of voltage gated $\mathrm{Na}^{+}$channels and some $\mathrm{K}^{+}$channels in order to regulate the current produced by the $\mathrm{Na}^{+}$channels. The nodes of Ranvier have almost the same structure in the CNS and in the peripheral nervous system (PNS), the most striking difference being that those situated at the PNS receive microvelli from the myelinating Schwann cells ensheathing the peripheral fibres. These microvelli have gliomedin protein with a capacity for clustering voltage gated $\mathrm{Na}^{+}$channels at the nodes of the peripheral fibres ${ }^{45,46}$. This is not the case for the nodes of Ranvier situated in the CNS myelinated fibres, which do not receive processes from the oligodendrocytes; but they do receive processes from the fibrous astrocytes whose function is unknown. However, knowing the capacity of the astrocytes for regulating the ionic concentration of the external medium, it is possible to assume that the process sent by the fibrous astrocytes to the nodes might buffer the concentration of $\mathrm{Na}^{+}$and $\mathrm{K}^{+}$ions within the medium to assure the successful transport of the APs along the fibre.

Therefore, it is possible to postulate that there is a coordinated action between the protoplasmic and fibrous astrocytes forming a net where the travelling $\mathrm{Ca}^{++}$waves propagate from one astrocyte to another through their GJs with the aim of attaining a successfully defined goal by adjusting the neuron and axon activities.

\section{ASTROCYTES IN PATHOLOGY}

Over the last decade, observations provided by several experimental studies have suggested that astrocytes play a key role in the onset and development of some primary degenerative disorders (PDD) of the $\mathrm{CNS}^{3}$. Most of those investigations have shown that astrocytes are the initial cells affected in those diseases, often followed by microglial activation.

Examples of this behaviour are as follows:

1) In frontotemporal dementia, astrocytes showing apoptotic signs are found at the onset of the disease when neuronal loss is still very rare, followed by astrocytosis and microgliosis ${ }^{47}$.

2) In Parkinson's disease, animal experimental evidence has demonstrated that $\alpha$-synuclein deposition in astrocytes initiates a non-cell autonomous killing of neurons through microglial signalling ${ }^{48}$.
3) The early and mid-term stages of Alzheimer's disease are marked by entorhinal atrophy of astroglia associated with synaptic disruption, altered neurotransmitter up-take and, finally, neuronal death due to excitotoxicity ${ }^{49}$.

4) In Huntington's disease, mice expressing mutated Huntingtin protein (mhtt) in neurons live normally or show only mild neurological signs, while mice expressing mhtt only in astrocytes develop neurological deficits and an earlier neuronal death ${ }^{50,51}$.

5) Regarding spinocerebellar ataxias, Garden et al..$^{52}$ and, later on, Custer et al. ${ }^{53}$ produced mice expressing ataxin-7 only in Bergmann glia, which was sufficient to bring on ataxia and neuronal degeneration.

In amyotrophic lateral sclerosis (ALS), astrogliosis and microgliosis are hallmarks of the illness all along its course $^{53}$. Other observations support the idea that an early primary astrocytic damage occurs in this disease ${ }^{2}$.

In $2011^{2}$ we proposed the possibility that ALS might be a prion disease due to a conformational change of a putative protein able to travel within the astrocytes' net devoted to motor neurons. Later, other authors also claimed that proteins such as TDP-43, Tau, $\alpha$-synuclein and SOD1 could acquire prion properties when mutated ${ }^{54,55,56}$.

A recent finding of Prusiner et al. ${ }^{57}$ further supports this concept. Studying multiple system atrophy characterized by the presence of glial cytoplasmic inclusions of $\alpha$-synuclein, they found that this protein behaved like a prion protein in mice expressing mutated $\alpha$-synuclein A53T producing neurodegeneration. The observations by Prusiner et al. are crucial because if this concept can be extrapolated to other neurodegenerative diseases we shall be much closer to the understanding of their pathogenesis. A possibility that could be envisaged is that the prion-like protein might be formed within the astrocytes travelling within the net they form, but that it may also enter neurons through the GJ connecting both cells.

In major depression, schizophrenia and bipolar illnesses there are subtle, though still weak, hints suggesting that astrocytic dysfunction plays a role in the pathogenesis of those entities. Astrocytic atrophy, decreased GFAP and reduced capacity for glutamate uptake and, therefore, diminished glutamine synthesis have been described ${ }^{58,59}$. With regard to cell loss there is much to be unveiled in these disorders; there is no definite agreement regarding the stability or loss of the number of astrocytes in these diseases ${ }^{60}$.

\section{FINAL REMARKS}

The findings commented on in this paper support the notion that astrocytes are probably executive cells within the CNS playing an important role in information processing, contributing to codifying messages within different neuronal-astrocytic nets. Also, it appears that astrocytes have a role in the onset 
and development of PDD of the CNS. A better understanding of their behaviour in these conditions will allow the acquisition of knowledge that will permit better understanding of the mechanisms underlying their pathogenesis. The constraints are that for those disorders there are no fully reliable animal models, as these diseases do not occur in wild type animals and are, therefore, restricted to the human species. Therefore, future work on the pathophysiology of PDD should be carried out on humans, rigorously honouring the human rights stated by international institutions controlling clinical research; notwithstanding that, experimental work will continue giving glimpses into particular aspects of those conditions.

\section{References}

1. Navarrete M, Araque A. The Cajal school and the physiological role of astrocytes: a way of thinking. Front Neuroanat. 2014;8:33-52. doi:10.3389/fnana.2014.00033

2. Sica RE, Nicola AF, Deniselle MC, Rodriguez G, Monachelli GM, Peralta LM et al. Sporadic amyotrophic lateral sclerosis: new hypothesis regarding its etiology and pathogenesis suggests that astrocytes might be the primary target hosting a still unknown external agent. Arq Neuropsiquiatr. 2011;69(4):699-706. doi:10.1590/S0004-282X2011000500023

3. Sica RE. Could astrocytes be the primary target of an offending agent causing the primary degenerative diseases of the human central nervous system? A hypothesis. Med Hypotheses. 2015;84(5):481-9. doi:10.1016/j.mehy.2015.02.004

4. Barnett SC, Linington C. Myelination: do astrocytes play a role? Neuroscientist. 2013;19(5):442-50. doi:10.1177/1073858412465655

5. Parpura V, Heneka MT, Montana V, Oliet SH, Schousboe A, Haydon PG et al. Glial cells in (patho) physiology.J Neurochem. 2012;121(1):4-27. doi:10.1111/j.1471-4159.2012.07664.x

6. DiNuzzo M, Mangia S, Maraviglia B, Giove F. Regulatory mechanisms for glycogenolysis and K+ uptake in brain astrocytes. Neurochem Int. 2013;63(5):458-64. doi:10.1016/j.neuint.2013.08.004

7. Pekny M, Pekna M. Astrocyte reactivity and reactive astrogliosis: costs and benefits. Physiol Rev. 2014;94(4):1077-98. doi:10.1152/physrev.00041.2013

8. Cregg JM, DePaul MA, Filous AR, Lang BT, Tran A, Silver J. Functional regeneration beyond the glial scar. Exp Neurol. 2014;253:197-207. doi:10.1016/j.expneurol.2013.12.024

9. Cahoy JD, Emery B, Kaushal A, Foo LC, Zamanian JL, Christopherson $\mathrm{KS}$ et al. A transcriptome database for astrocytes, neurons and oligodendrocytes: a new resource for understanding brain development and function. J Neurosci. 2008;28(1):264-78. doi:10.1523/JNEUROSCI.4178-07.2008

10. Angelova PR, Kasymov V, Christie I, Sheikhbahaei S, Turovsky E, Marina $\mathrm{N}$ et al. Functional oxygen sensitivity of astrocytes.J Neurosci. 2015;35(29):10460-73. doi:10.1523/ JNEUROSCI.0045-15.2015

11. Duan CL, Liu CW, Shen SW, Yu Z, Mo JL, Chen XH et al. Striatal astrocytes transdifferentiate into functional mature neurons following ischemic brain injury. Glia. 2015;63(9):1660-70. doi:10.1002/glia.22837

12. Howarth C, Gleeson P, Attwell D. Updated energy budgets for neural computation in the neocortex and cerebellum.J Cereb Blood Flow Metab. 2012;32(7):1222-32. doi:10.1038/jcbfm.2012.35

13. Winship IR, Plaa N, Murphy TH. Rapid astrocyte calcium signals correlate with neuronal activity and onset of the hemodynamic response in vivo. J Neurosci. 2007;27(23):6268-72. doi:10.1523/ JNEUROSCI.4801-06.2007

14. Nedergaard M, Verkhratsky A. Artifact versus reality: how astrocytes contribute to synaptic events. Glia. 2012;60(7):1013-23. doi:10.1002/glia.22288

15. Panov A, Orynbayeva Z, Vavilin V, Lyakhovich V. Fatty acids in energy metabolism of the central nervous system. Biomed Res Int. 2014;2014:472459 doi:10.1155/2014/472459.
16. Eugenin EA, Basilio D, Sáez JC, Orellana JA, Raine CS, Bukauskas $F$ et al. The role of gap junction channels during physiologic and pathologic conditions of the human central nervous system. J Neuroimmune Pharmacol. 2012;7(3):499-518. doi:10.1007/s11481-012-9352-5

17. Pirttimaki TM, Parri HR. Astrocyte plasticity: implications for synaptic and neuronal activity. Neuroscientist. 2013;19(6):604-15. doi:10.1177/1073858413504999

18. Friede R. Der quantitative Anteil der Glia an der Cortexentwicklung. Acta Anat (Base). 1954;20(3):290-6. doi:10.1159/000140905

19. Kast B. The best supporting actors. Nature. 2001;412(6848):674-6. doi:10.1038/35089223

20. Hawkins A, Olszewski J. Glia/nerve cell index for cortex of the whale. Science. 1957;126(3263):76-7. doi:10.1126/science.126.3263.76

21. Tower DB, Elliot KA. Activity of acetylcholine system in cerebral cortex of various unanesthetized mammals. Am J Physiol. 1952;168(3):747-59.

22. Tower DB. Structural and functional organization of mammalian cerebral cortex: the correlation of neurone density with brain size; cortical neurone density in the fin whale (Balaenoptera physalus $L$ ) with a note on the cortical neurone density in the Indian elephant. $J$ Comp Neurol. 1954;101(1):19-51. doi:10.1002/cne.901010103

23. Herculano-Houzel S. The glia/neuron ratio: how it varies uniformly across brain structures and species and what that means for brain physiology and evolution. Glia. 2014;62(9):1377-91. doi:10.1002/glia.22683

24. Pelvig DP, Pakkenberg H, Stark AK, Pakkenberg B. Neocortical glial cell numbers in human brains. Neurobiol Aging. 2008;29(11):1754-62. doi:10.1016/j.neurobiolaging.2007.04.013

25. Belmonte JC, Callaway EM, Caddick SJ, Churchland P, Feng G, Homanics GE et al. Brains, genes, and primates. Neuron. 2015;86(3):617-31. doi:10.1016/j.neuron.2015.03.021

26. Nash B, loannidou K, Barnett SC. Astrocyte phenotypes and their relationship to myelination. J Anat. 2011;219(1):44-52. doi:10.1111/j.1469-7580.2010.01330.x

27. Pérez-Alvarez A, Araque A. Astrocyte-neuron interaction at tripartite synapses. Curr Drug Targets. 2013;14(11):1220-4. doi:10.2174/13894501113149990203

28. Richards GS, Simionato E, Perron M, Adamska M, Vervoort M, Degnan BM. Sponge genes provide new insight into the evolutionary origin of the neurogenic circuit. Curr Biol. 2008;18(15):1156-61. doi:10.1016/j.cub.2008.06.074

29. Sakarya O, Armstrong KA, Adamska M, Adamski M, Wang IF, Tidor B et al. A post-synaptic scaffold at the origin of the animal kingdom. PLoS ONE. 2007;2(6):e506. doi:10.1371/journal.pone.0000506

30. Krubitzer L. In search of a unifying theory of complex brain evolution. Ann N Y Acad Sci. 2009;1156(1):44-67. doi:10.1111/j.1749-6632.2009.04421.x

31. Perea G, Sur M, Araque A. Neuron-glia networks: integral gear of brain function. Front Cell Neurosci. 2014;8:378. doi:10.3389/fncel.2014.00378

32. Hooks BM, Chen C. Critical periods in the visual system: changing views for a model of experience- dependent plasticity. Neuron. 2007;56(2):312-26. doi:10.1016/j.neuron.2007.10.003 
33. Bruner E. Geometric morphometrics and paleoneurology: brain shape evolution in the genus Homo. J Hum Evol. 2004;47(5):279-303. doi:10.1016/j.jhevol.2004.03.009

34. Emes RD, Pocklington AJ, Anderson CNG, Bayes A, Collins $\mathrm{MO}$, Vickers CA et al. Evolutionary expansion and anatomical specialization of synapse proteome complexity. Nat Neurosci. 2008;11(7):799-806. doi:10.1038/nn.2135

35. Parpura $V$, Verkhratsky $A$. The astrocyte excitability brief: from receptors to gliotransmission. Neurochem Int. 2012;61(4):610-21. doi:10.1016/j.neuint.2011.12.001

36. Halassa MM, Fellin T, Haydon PG. The tripartite synapse: roles for gliotransmission in health and disease. Trends Mol Med. 2007;13(2):54-63. doi:10.1016/j.molmed.2006.12.005

37. Fellin T, Pascual O, Gobbo S, Pozzan T, Haydon PG, Carmignoto G. Neuronal synchrony mediated by astrocytic glutamate through activation of extrasynaptic NMDA receptors. Neuron. 2004;43(5):72943. doi:10.1016/j.neuron.2004.08.011

38. Zhang JM, Wang HK, Ye CQ, Ge W, Chen Y, Jiang ZL et al. ATP released by astrocytes mediates glutamatergic activity-dependent heterosynaptic suppression. Neuron. 2003;40(5):971-82. doi:10.1016/S0896-6273(03)00717-7

39. Nag S. Morphology and properties of astrocytes. Methods Mol Biol. 2011;686:69-100. doi:10.1007/978-1-60761-938-3_3

40. Hewett JA. Determinants of regional and local diversity within the astroglial lineage of the normal central nervous system. J Neurochem. 2009;110(6):1717-36. doi:10.1111/j.1471-4159.2009.06288.x

41. Martín R, Bajo-Grañeras R, Moratalla R, Perea G, Araque A Circuit specific signaling in astrocyte-neuron networks in basal ganglio pathways. Science. 2015;349(6249):730-4. doi:10.1126/science.aaa7945

42. Colombo JA, Reisin HD. Interlaminar astroglia of the cerebral cortex: a marker of the primate brain. Brain Res. 2004;1006(1):126-31. doi:10.1016/j.brainres.2004.02.003

43. Halassa MM, Fellin T, Takano H, Dong JH, Haydon PG. Synaptic islands defined by the territory of a single astrocyte. J Neurosci. 2007;27(24):6473-7. doi:10.1523/JNEUROSCI.1419-07.2007

44. Jourdain P, Bergersen LH, Bhaukaurally K, Bezzi P, Santello M, Domerca $M$ et al. Glutamate exocytosis from astrocytes controls synaptic strength. Nat Neurosci. 2007;10(3):331-9. doi:10.1038/nn1849

45. Martin PM, Cifuentes-Diaz C, Garcia M, Goutebroze L, Girault JA. [Axon and Schwann cells... so far away, so close]. Rev Neurol (Paris). 2008;164(12):1057-62. doi:10.1016/j.neurol.2008.10.003

46. Salzer Jl, Brophy PJ, Peles E. Molecular domains of myelinated axons in the peripheral nervous system. Glia. 2008;56(14):1532-40. doi:10.1002/glia.20750

47. Broe M, Kril J, Halliday GM. Astrocytic degeneration relates to the severity of disease in frontotemporal dementia. Brain. 2004;127(10):2214-20. doi:10.1093/brain/awh250
48. Halliday GM, Stevens CH. Glia: initiators and progressors of pathology in Parkinson's disease. Mov Disord. 2011;26(1):6-17. doi:10.1002/mds.23455

49. Olabarria M, Noristani HN, Verkhratsky A, Rodríguez JJ. Concomitant astroglial atrophy and astrogliosis in a triple transgenic animal model of Alzheimer's disease. Glia. 2010;58(7):831-8. doi:10.1002/glia.20967

50. Bradford J, Shin JY, Roberts M, Wang CE, Li XJ, Li S. Expression of mutant huntingtin in mouse brain astrocytes causes agedependent neurological symptoms. Proc Natl Acad Sci USA. 2009:106(52):22480-5. doi:10.1073/pnas.0911503106

51. Faideau M, Kim J, Cormier K, Gilmore R, Welch M, Auregan G et al. In vivo expression of polyglutamine-expanded huntingtin by mouse striatal astrocytes impairs glutamate transport: a correlation with Huntington's disease subjects. Hum Mol Genet. 2010;19(15):3053-67. doi:10.1093/hmg/ddq212

52. Garden GA, Libby RT, Fu YH, Kinoshita Y, Huang J, Possin DE et al. Polyglutamine-expanded ataxin-7 promotes non-cell-autonomous purkinje cell degeneration and displays proteolytic cleavage in ataxic transgenic mice.J Neurosci. 2002;22(12):4897-905.

53. Custer SK, Garden GA, Gill N, Rueb U, Libby RT, Schultz C et al. Bergmann glia expression of polyglutamine-expanded ataxin-7 produces neurodegeneration by impairing glutamate transport. Nat Neurosci. 2006;9(10)1302-11. doi:10.1038/nn1750

54. Papadeas ST, Kraig SE, O'Banion C, Lepore AC, Maragakis NJ. Astrocytes carrying the superoxide dismutase 1(SOD1G93A) mutation induce wild type motor neuron degeneration in vivo. Proc Natl Acad Sci USA. 2011;108(43):17803-8. doi:10.1073/pnas.1103141108

55. Tong J, Huang C, Bi F, Wu Q, Huang B, Liu X et al. Expression of ALS-linked TDP-43 mutant in astrocytes causes noncell-autonomous motor neuron death in rats. EMBO J. 2013;32(13):1917-26. doi:10.1038/emboj.2013.122

56. Grad LI, Fernando SM, Cashman NR. From molecule to molecule and cell to cell: prion-like mechanisms in amyotrophic lateral sclerosis. Neurobiol Dis. 2015;77:257-65. doi:10.1016/j.nbd.2015.02.009

57. Prusiner SB, Woerman AL, Mordes DA et al. Evidence for $\alpha$-synuclein prions causing multiple system atrophy in humans with parkinsonism. Proc Natl Acad Sci U S A. 2015; pii: 201514475. doi:

58. Rajkowska G, Miguel-Hidalgo JJ. Gliogenesis and glial pathology in depression. CNS Neurol Disord Drug Targets. 2007;6(3):219-33. doi:10.2174/187152707780619326

59. Hercher C, Chopra V, Beasley CL. Evidence for morphological alterations in prefrontal white matter glia in schizophrenia and bipolar disorder. J Psychiatry Neurosci. 2014;39(6):376-85. doi:10.1503/jpn.130277

60. Verkhratsky A, Parpura V. Astrogliopathology in neurological, neurodevelopmental and psychiatric disorders. Neurobiol Dis. 2015;85:254-61. doi:10.1016/j.nbd.2015.03.025 